\title{
CUTLASS/IMAGE observations of high-latitude convection features during substorms
}

\author{
T. K. Yeoman ${ }^{1}$, H. Lühr ${ }^{2}$ \\ ${ }^{1}$ Department of Physics and Astronomy, University of Leicester, University Road, Leicester, LE1 7RH, UK \\ ${ }^{2}$ GeoForschungsZentrum Postdam, Telegrafenberg, D-14473 Postdam, Germany
}

Received: 18 September 1996 / Revised: 24 January 1997 / Accepted 10 February 1997

\begin{abstract}
The CUTLASS Finland HF radar has been operational since February 1995. The radar frequently observes backscatter during the midnight sector from a latitude range $70-75^{\circ}$ geographic, latitudes often associated with the polar cap. These intervals of backscatter occur during intervals of substorm activity, predominantly in periods of relatively quiet magnetospheric activity, with $K_{p}$ during the interval under study being 2and $\Sigma K_{p}$ for the day being only 8-. During August 1995 the radar ran in a high time resolution mode, allowing measurements of line-of-sight convection velocities along a single beam with a temporal resolution of $14 \mathrm{~s}$, and measurement of a full spatial scan of line-ofsight convection velocities every four minutes. Data from such scans reveal the radar to be measuring return flow convection during the interval of substorm activity. For three intervals during the period under study, a reduction in the spatial extent of radar backscatter occurred. This is a consequence of $D$ region $\mathrm{HF}$ absorption and its limited extent in the present study is probably a consequence of the high latitude of the substorm activity, with the electrojet centre lying between $67^{\circ}$ and $71^{\circ}$ geomagnetic latitude. The high time resolution beam of the radar additionally demonstrates that the convection is highly time dependent. Pulses of equatorward flow exceeding $\sim 600 \mathrm{~m} \mathrm{~s}^{-1}$ are observed with a duration of $\sim 5 \mathrm{~min}$ and a repetition period of $\sim 8 \mathrm{~min}$. Their spatial extent in the CUTLASS field of view was $400-500 \mathrm{~km}$ in longitude, and 300 $400 \mathrm{~km}$ in latitude. Each pulse of enhanced equatorward flow was preceded by an interval of suppressed flow and enhanced ionospheric Hall conductance. The transient features are interpreted as being due to ionospheric current vortices associated with field aligned current pairs. The relationship between these observations and substorm phenomena in the magnetotail is discussed.
\end{abstract}

Correspondence to: T. K. Yeoman

\section{Introduction}

Substorm physics remains a very active field and in spite of recent progress towards an understanding of the substorm process, that understanding is as yet incomplete. The magnetospheric processes which lead to the expansion phase onset, the location of this onset region and the relationship between the possible magnetospheric source regions and the ionospheric phenomena involved in substorm breakup are still a matter for debate. A topic of particular interest is the spatial and temporal development of the ionospheric conductivities and electric field in the auroral electrojets, which are known to be highly time-dependent (e.g. Kirkwood et al., 1988) and which combine to produce the characteristic ground magnetic perturbations during the substorm expansion phase. Localised electrojet features in which ionospheric flows were suppressed to values as low as $50 \mathrm{~m} \mathrm{~s}^{-1}$ and accelerated up to values as high as $1 \mathrm{~km} \mathrm{~s}^{-1}$ have been observed within a few minutes, accompanied by conductance variations between 20 and 100 S (Morelli et al., 1995), whilst Yeoman and Pinnock (1996) observed the evolution of the overall convection pattern on a time scale of only $\sim 10 \mathrm{~min}$. This study exploits for the first time the potential for HF radars to make very high time resolution (14 s) measurements of the ionospheric plasma convection velocity during the highly dynamic substorm expansion phase and relates these signatures to the local ground magnetic field measurements. The HF radar technique is particularly useful in the high conductivity, low electric field region of the ionospheric location of substorm onset where a drop out in $\mathrm{E}$ region backscatter, indicative of low flow velocity (a consequence of VHF $\mathrm{E}$ region radar instrumental threshold effects, see, e.g. Robinson, 1986), has hampered previous VHF radar studies (Baumjohann et al., 1981; Inhester et al., 1981; Opgenoorth et al., 1983). However, substorm-associated HF absorption has, in the past, limited the use of HF radars to a small 
number of studies where extensive regions of velocity measurements were possible (Morelli et al., 1995; Yeoman and Pinnock, 1996).

In the present study an analysis of HF radar measurements of ionospheric convection and the location and dynamics of the auroral electrojets from ground magnetometers is presented. This demonstrates that for intervals of quiet magnetic activity, when the main substorm electrojets are at high latitudes $\left(\sim 70^{\circ}\right.$ geomagnetic), HF absorption has only a limited effect on the radar observations of the substorm expansion phase. Furthermore these observations provide a context for data from the high time resolution beam of the CUTLASS Finland radar, which has revealed new observations of transient convection vortices in the substorm expansion phase. These vortices are characterised with the radar and magnetometer data and their relationship with magnetotail phenomena is discussed.

\section{Instrumentation}

The ionospheric convection velocities in this study are provided by the Finland radar of the Co-operative UK Twin-Located Auroral Sounding System (CUTLASS). CUTLASS is a bistatic HF coherent radar, with stations in Finland and Iceland, and forms part of the international SuperDARN chain of HF radars (Greenwald et al., 1995). Each radar of the system is a frequency agile (8-20 MHz) radar, routinely measuring the line-ofsight (1-o-s) Doppler velocity and spectral width of, and the backscattered power from, ionospheric plasma irregularities. The radars form 16 beams of separation $3.24^{\circ}$. Each beam is gated into 75 range bins, each of length $45 \mathrm{~km}$ in standard operations. During standard operations the dwell time for each beam is $7 \mathrm{~s}$, giving a full 16 beam scan, covering $52^{\circ}$ in azimuth and over $3000 \mathrm{~km}$ in range (an area of over $4 \times 10^{6} \mathrm{~km}^{2}$ ), every 2 min. Common-volume data from the two stations can be combined to provide convection velocities perpendicular to the magnetic field, although the interval under study here occurred before the completion of the Iceland radar. For this interval the Finland radar was operating a non-standard scan mode. In this mode, rather than the usual anticlockwise sweep through beams $15,14,13, \ldots, 0$ the sequence $15,9,14,9,13,9, \ldots, 1,9,0,9$ was employed. This allows the construction of full 16 beam scans at a reduced temporal resolution of $4 \mathrm{~min}$, in addition to the provision of high time resolution (14 s) data along a single look direction (in this case beam 9, a beam which approximately overlies the main meridional chain of the IMAGE array). Such radar modes were first used by Pinnock et al., (1995) for studies of the ionospheric footprint of the cusp. This dataset is supplemented here by data from ground magnetometers, provided by stations from two arrays, the UK SubAuroral Magnetometer Network (SAMNET) (Yeoman et al. 1990), and the International Monitor for Auroral Geomagnetic Effects, IMAGE) (Lühr, 1994). The instruments are fluxgate magnetometers. SAMNET has a sampling interval of $5 \mathrm{~s}$ and the IMAGE array has $10 \mathrm{~s}$ sampling for the interval under consideration here. The IMAGE data is presented in $X, Y, Z$ coordinates, where $X$ is geographic northwards, $Y$ geographic eastwards and $Z$ is vertically downwards. All data have had a quiet day mean subtracted. The field-of-view of the CUTLASS Finland radar, together with the locations of the ground magnetometers used in this study, are illustrated in Fig. 1.

\section{Observations}

Figure 2 presents the $X$ and $Z$ component magnetic field measurements from a subset of the IMAGE array, forming a meridional chain from Svalbard to northern Scandinavia. Mid-latitude Pi2 measurements from both the IMAGE and SAMNET arrays (OUJ only shown, filtered between 200 and $20 \mathrm{~s}$, bottom panels of Fig. 2) have been used to provide a reference onset time for the substorm onsets/intensifications studied in this work (Rostoker et al., 1980). The times of these mid-latitude Pi2s for the eight substorm events identified by this

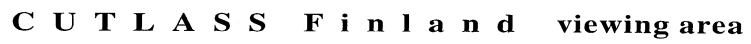

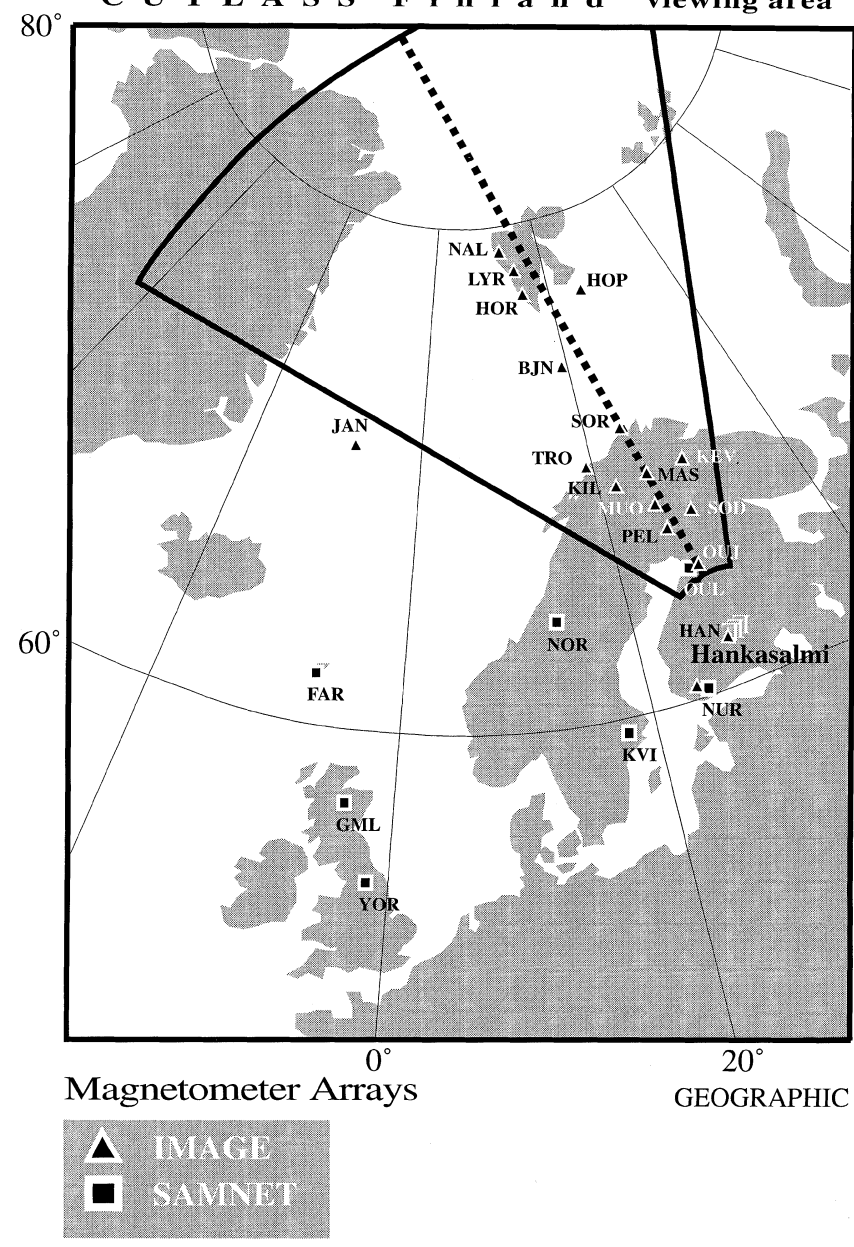

Fig. 1. Locations of the field-of-view of the CUTLASS Finland radar together with the IMAGE and SAMNET magnetometer stations used in this study. Beam 9 of CUTLASS Finland, which is sampled at a high rate, is marked by a dashed line 


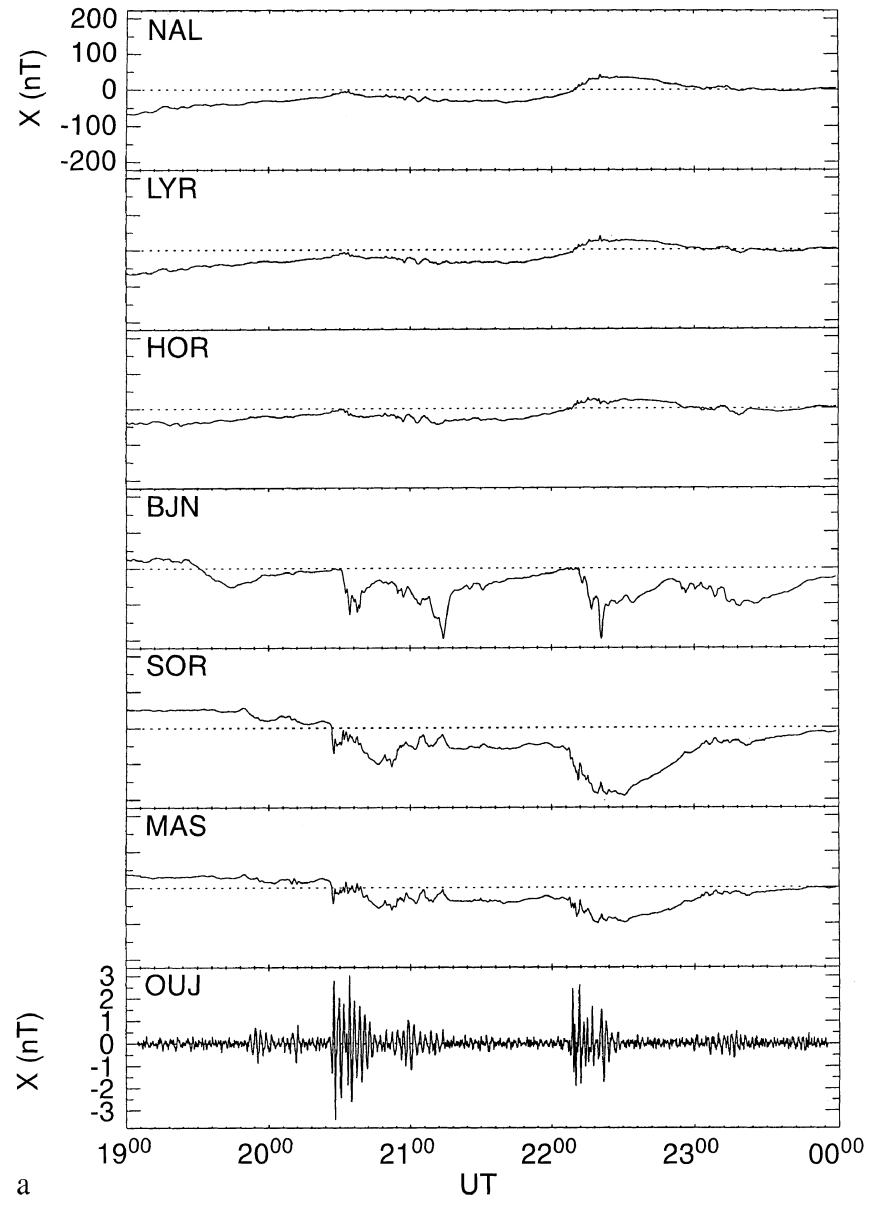

Fig. 2a,b. Ground magnetograms from the IMAGE array a $X$ components, $\mathbf{b} Z$ components. A subset of the IMAGE array, forming a meridional chain from Svalbard to northern Scandinavia, is

mechanism are marked in Fig. 2 by vertical lines and are ( \pm 1 minute): 19:52, 20:08, 20:26, 20:33, 20:57, 22:08, 22:19, and 22:54 UT.

\subsection{Ground-based magnetic response}

The mid-latitude $X$ and $Y$ component magnetic field data from the SAMNET array, as well as timing the substorm onset/intensification, have been utilised here to determine the longitudinal position of the substorm current wedge (SCW) for each onset/intensification (see e.g. Lester et al., 1983; Yeoman et al., 1991). The north, X, and vertical, $Z$, components of the magnetic field measured by the higher latitude IMAGE stations can be employed in a similar fashion to the SAMNET $X$ and $Y$ component data, to provide information on the location of the substorm-enhanced westward electrojet (e.g. Kisabeth and Rostoker, 1973; Samson and Rostoker, 1983) and hence the latitude of the centre of the electrojet. The high-latitude stations can also give an indication of the longitude of the westward travelling surge.

The mid-latitude magnetometer bay structure (not shown) provides the following picture of the longitudi-

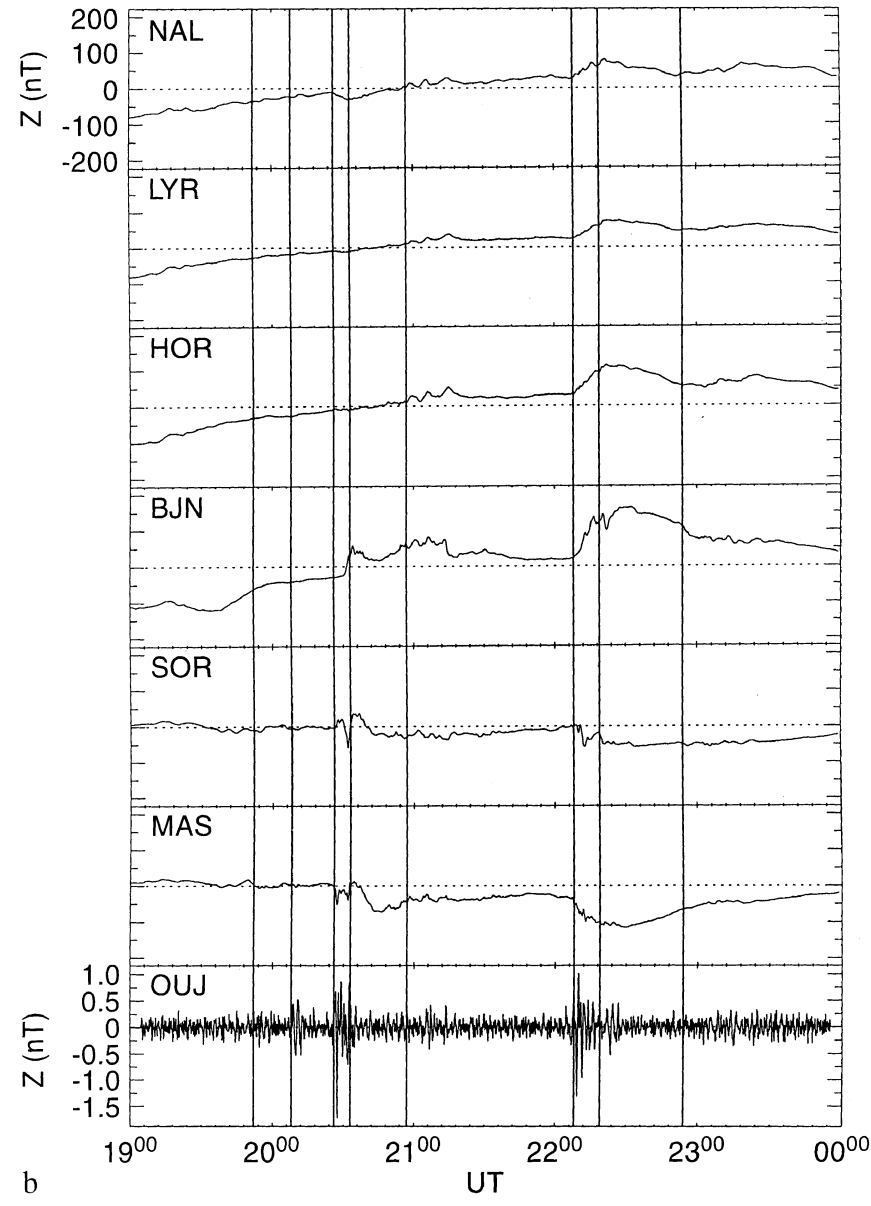

displayed. A bandpass filtered (200 - $20 \mathrm{~s})$ trace from OUJ, highlighting $\mathrm{Pi} 2$ pulsation activity, is included in the bottom panel. Substorm expansion phase onset/intensifications are marked with vertical lines

nal development of the substorms during this interval. At 19:52 UT a broad SCW was observed the location of which, relative to the ground instrumentation, is unclear. At 20:08 UT another substorm onset was observed to the east of the ground magnetometers. Neither of these onsets was centred near auroral zone magnetometers displayed in Fig. 2, and no strong current systems are observed local to these magnetometer stations. At 20:26 UT a stronger substorm expansion phase onset signature was observed. The SCW for this event was centred east of the magnetometer arrays, with the upward field aligned current (FAC) between the longitudes of KVI and NUR. An intensification of this substorm occurred at 20:33 UT. Both these onsets had magnetic signatures of moderately strong current systems near the auroral zone instrumentation. Subsequent to this a small substorm signature was observed west of the instrumentation at 20:57 UT. Stronger substorm activity was observed later at 22:08 UT. The SCW for this event was centred over the longitude of NOR with the upward FAC to the west of the instrumentation. There was a further intensification of this event at 22:19 UT. The final substorm event in the interval is a distant event to the west of the instrumentation at 22:54 UT. 
The IMAGE magnetograms in Fig. 2 reveal clear magnetic field perturbations due to local auroral substorm current systems for onsets, 3, 4, 6 and 7. At 20:26 UT a substorm-enhanced westward electrojet centred between MAS and SOR is clearly visible in the $X$ and $Z$ component magnetic field. This is coincident with mid-latitude magnetometer observations of an upward FAC just to the west of the IMAGE stations of Svalbard. The largest electrojet signature observed in the $X$ component for this onset is at SOR. Seven minutes later an intensification of this expansion phase onset is observed, at 20:33 UT. This intensification sees the electrojet centre, deduced from the strongest negative $X$ component perturbation and the positive $Z$ component perturbation of the magnetic field at $\mathrm{BJN}$, move poleward to just south of BJN. This onset has also expanded to the west. The transient $Z$ component observed at SOR at this time is most probably due to coastal induction effects. The substorm onset indicated by $\mathrm{Pi} 2$ activity at 20:56 UT is not very clear in the IMAGE magnetograms. At JAN, however, $25^{\circ}$ to the west, the expected decrease in the $X$ component is observed. The westward electrojet to the east at BJN appears to intensify only after 21:05 UT, and it is this intensification which will be at the centre of the subsequent study. The substorm onset at 22:08 UT is centred between the latitudes of SOR and BJN, with the largest auroral zone signatures in fact being observed to the west of these stations at JAN. The centre of the intensification at 22:19 UT moves north and east, close to $\mathrm{BJN}$.

\subsection{Radar measurements of ionospheric convection}

Data from the 4-min full 16-beam scans of the radar (not shown) reveal the following overall pattern: prior to $\sim 20: 30$ UT the west-pointing beams of the radar were observing line-of-sight flow away from the radar and the east-pointing beams, flow toward the radar. Such flows are indicative of return flow in the dusk convection cell. From 20:30 UT through to 22:30 UT flow across the entire field-of-view of the radar was toward the radar, indicative that the radar was in a region of equatorward flow in the convection reversal region between the two main cells of return flow. The flow during these times was fairly slow, not in general exceeding $200 \mathrm{~m} \mathrm{~s}^{-1}$. After 22:30 UT a pattern of flow toward the radar in the westpointing beams and line of sight flow away from the radar in the east-pointing beams was observed, indicating that the radar had moved into the return flow region of the dawn convection cell.

A summary of the convection velocity response to the interval of substorm activity is given in Fig. 3a. This figure presents the CUTLASS high time resolution lineof-sight Doppler velocity measurements from beam 9 from 19:00-24:00 UT. The line-of-sight velocity is displayed as a function of time and geomagnetic latitude (throughout this study altitude adjusted corrected geomagnetic, or AACGM, coordinates, based on Baker and Wing, 1989 are employed). A colour scale representation is used, with flow toward the radar (equatorward flow, positive Doppler velocity) plotted in blue and flow away from the radar (poleward flow, negative Doppler velocity) plotted in red. Beam 9 is the high time resolution beam which most closely overlies the meridional magnetometer chain displayed in Fig. 2, and is illustrated as a dashed line in Fig. 1. The line-of-sight velocity in beam 9 is toward the radar from 19:00-23:20 UT, indicative of the dusk sector return flow and equatorward flow described in the preceding paragraph. After 23:20 UT flow is predominantly away from the radar as the radar enters the dawn return flow convection cell.

One clear substorm-associated signature in the HF radar data in Fig. $3 \mathrm{a}$ are the periods of reduced backscatter in the intervals 20:07-20:12 UT, 20:2720:44 UT and 22:02-22:24 UT. These "dropouts" in radar backscatter are clearly correlated with five of the substorm onsets, and smaller dropouts may be correlated with the other three. The first of these dropouts in backscatter is observed at the same time as a substorm onset, the mid-latitude magnetic signature of which places it to the east of beam 9 of the radar. The second, and largest, is observed during the substorm onset and subsequent intensification which occurred close to the auroral instrumentation, although centred to the east, at 20:26 UT and 20:33 UT respectively. The final dropout occurred during the later substorm event which was again nearby the ground instrumentation but this time centred to the west. In spite of these dropouts, the CUTLASS Finland radar records some backscatter throughout the interval of substorm activity, and a substantial band of backscatter, extending from $65^{\circ}-73^{\circ}$ geomagnetic, some $890 \mathrm{~km}$, is observed for the majority of the interval under study. The minimum coverage of the radar occurs at 20:33 UT, when the data is reduced to a narrow band of $2^{\circ}(\sim 220 \mathrm{~km})$ between $65^{\circ}$ and $67^{\circ}$ geomagnetic latitude.

\subsection{Expansion phase convection}

In addition to the overall ionospheric convection and HF absorption features associated with the substorm process outlined in the previous section, a number of very dynamic features are observed in the geomagnetic field between 20:50 and 21:40 UT. In Fig. 2 these can be seen most clearly in the $X$ component magnetic field at BJN. The magnetic signatures of these dynamic features in the $X$ and $Y$ component at BJN and SOR are presented in more detail in Fig. 4, along with the line of sight velocity from beam 9, range gate 25 of the CUTLASS Finland radar, which lies close to overhead of BJN. In spite of the expansion phase onset and intensification at 20:27 and 20:33 UT, which have resulted in a restriction of HF backscatter in the interval 20:27-20:44 UT, measurements of ionospheric velocity are available from $8^{\circ}$ of magnetic latitude after that time. In Fig. 4 the magnetic signatures can clearly be seen to be accompanied by dynamic convection velocity measurements. The equatorward velocity pulses mea- 


\section{SUPERDARN / IMAGE 6 August 1995}

a FINLAND: vel

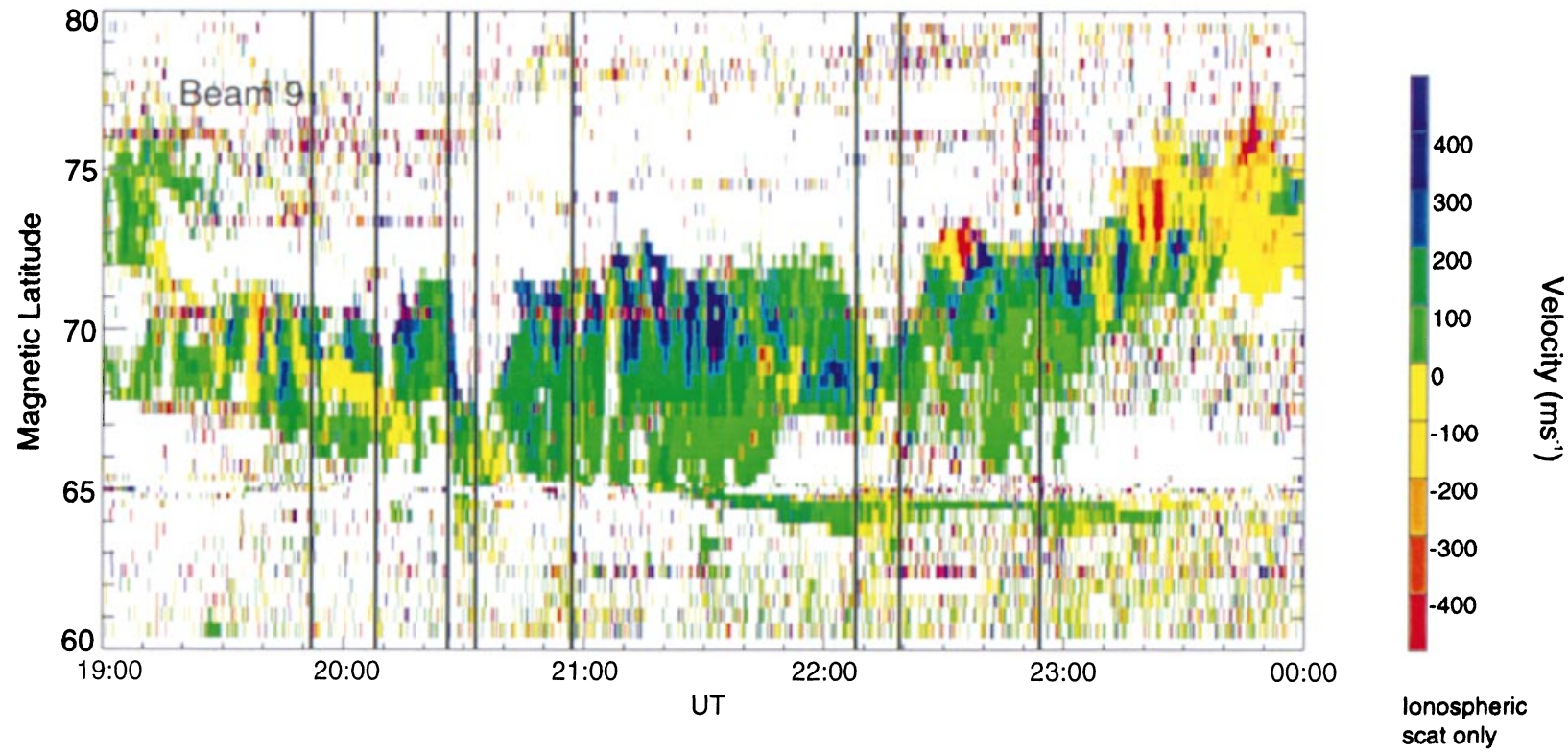

b

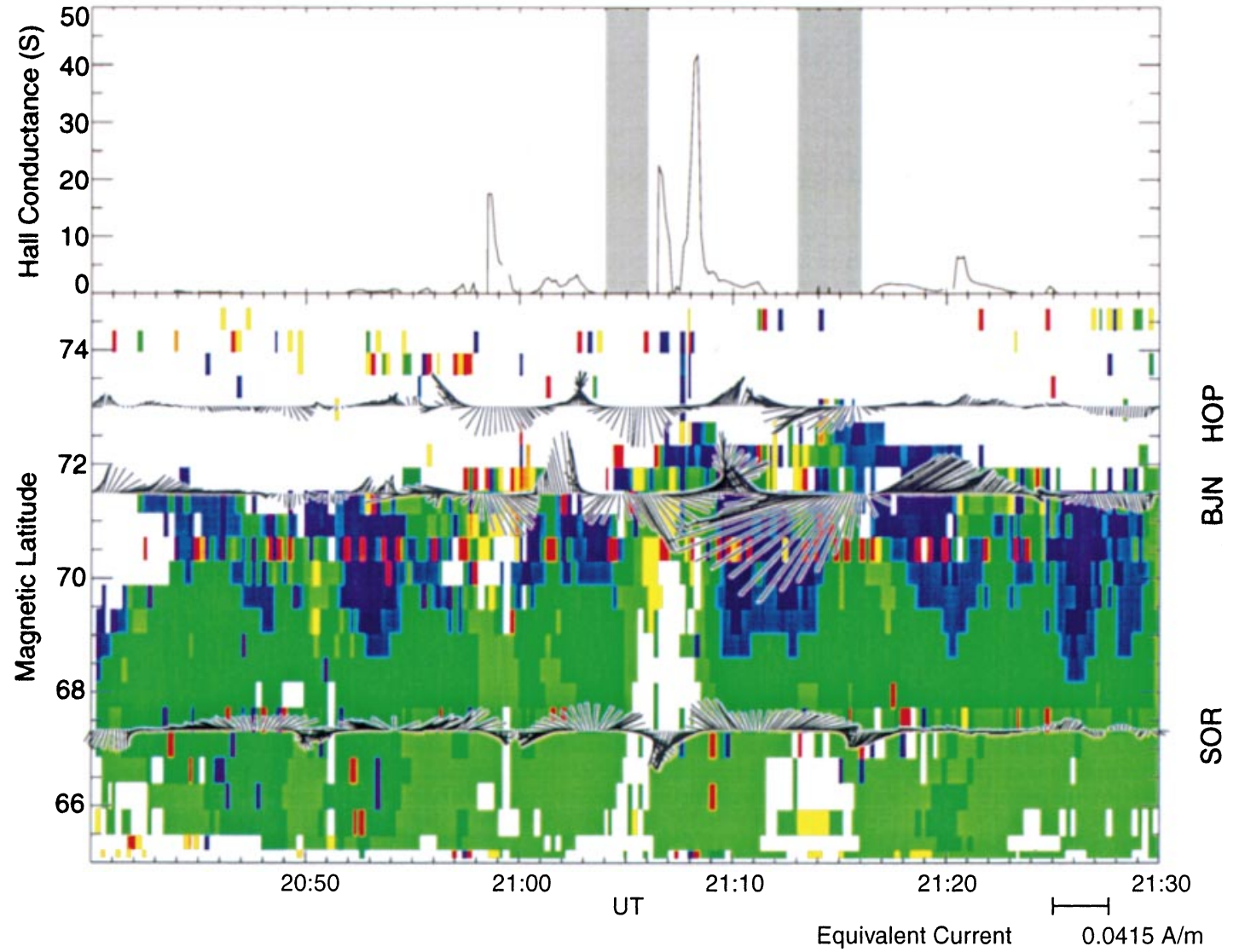

Fig. 3. a Line-of-sight velocity from beam 9 of the CUTLASS Finland radar, as a function of magnetic latitude and time, showing dynamic convection velocity features and intervals of substormassociated HF absorption. Substorm expansion phase onsets/intensifications are marked with vertical lines. Positive Doppler velocities are towards the radar (equatorwards) and negative Doppler velocities are away from the radar (polewards); b CUTLASS Finland line-of-sight velocities as a function of magnetic latitude and time. The equivalent current intensities derived from the magnetic field data from SOR, $\mathrm{BJN}$ and HOP are overlaid at the magnetic latitude appropriate for each station. The upper panel presents an estimate of the Hall conductance, derived from the BJN magnetic field and the line-ofsight velocity from the overlying range gate (see text for details) 


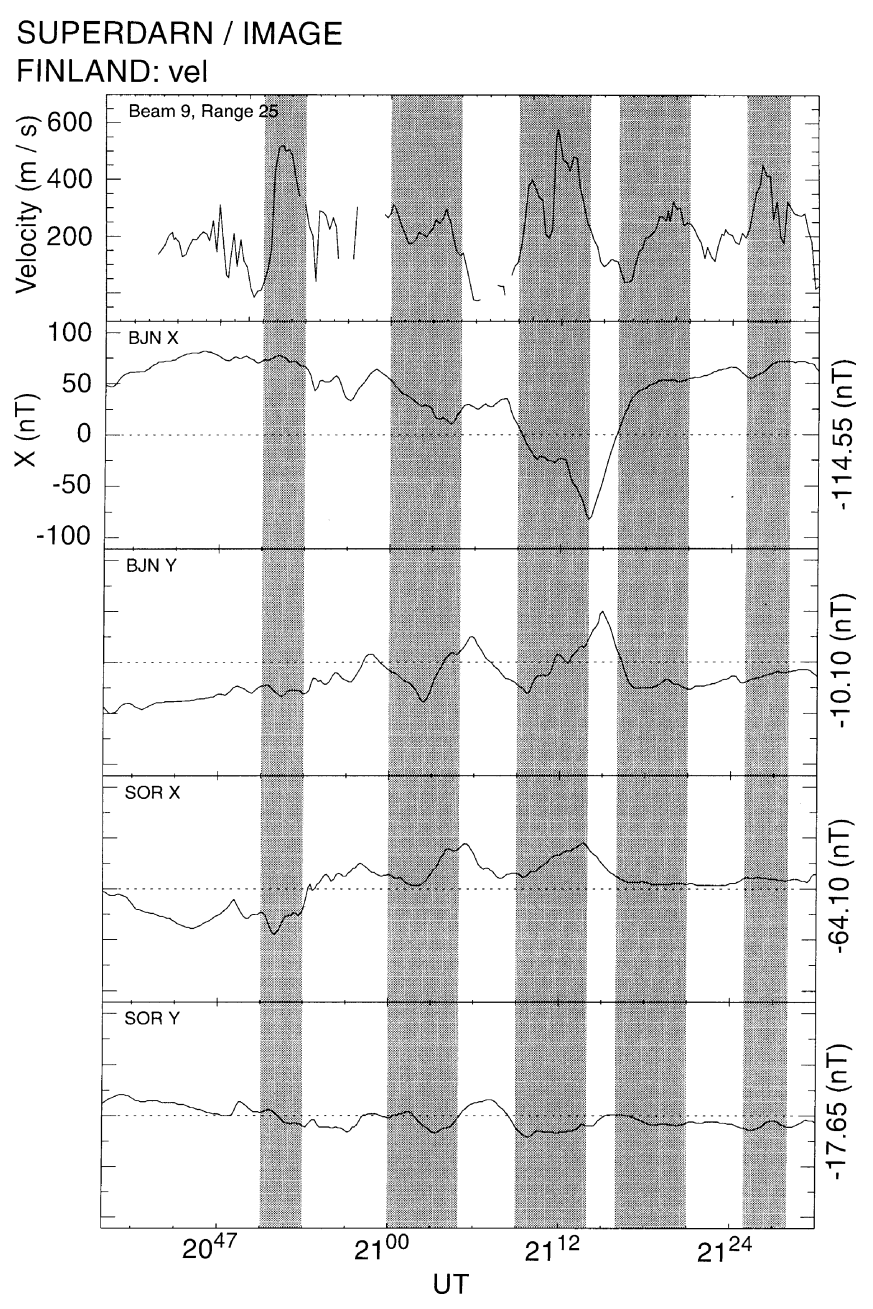

Fig. 4. Line of sight velocity data from beam 9 , range gate 25 of the CUTLASS Finland radar (top panel) which approximately overlies BJN, and the $X$ and $Y$ components of the magnetic field measured at BJN and SOR. The times of equatorward velocity pulses are marked by shaded bars

sured by CUTLASS are marked in the figure by shaded bars. In Fig. 4 it is apparent that, for the central three velocity perturbations at least, negative perturbations in the $Y$ component at $\mathrm{BJN}$ are associated with pulses of strong equatorward flow measured in beam 9 of the CUTLASS Finland radar, although the magnetic signatures lead the flow signatures by $\sim 70 \mathrm{~s}$. This offset is discussed in more detail in Sect. 4.2. There is evidence of slight negative $Y$ perturbations in the first and last velocity pulses. These pulses are typically of $5 \mathrm{~min}$ duration, with an inter-pulse interval of about $3 \mathrm{~min}$. They peak at a line-of-sight flow of $\sim 600 \mathrm{~m} \mathrm{~s}^{-1}$. The spatial extent of these pulses can be measured using the 4-min resolution, 16 beam scan of the CUTLASS radar. Care needs to be taken with this data, as the duration of the scan is comparable with the duration of the velocity pulses. In Fig. 5, however, the scan from 21:10:17 to 21:14:17 UT is displayed. A greyscale representation is used, with flow toward the radar plotted in dark shading and flow away from the radar plotted in light shading. The whole of this scan occurred during the third

\section{SUPERDARN PARAMETER PLOT \\ FINLAND: vel}

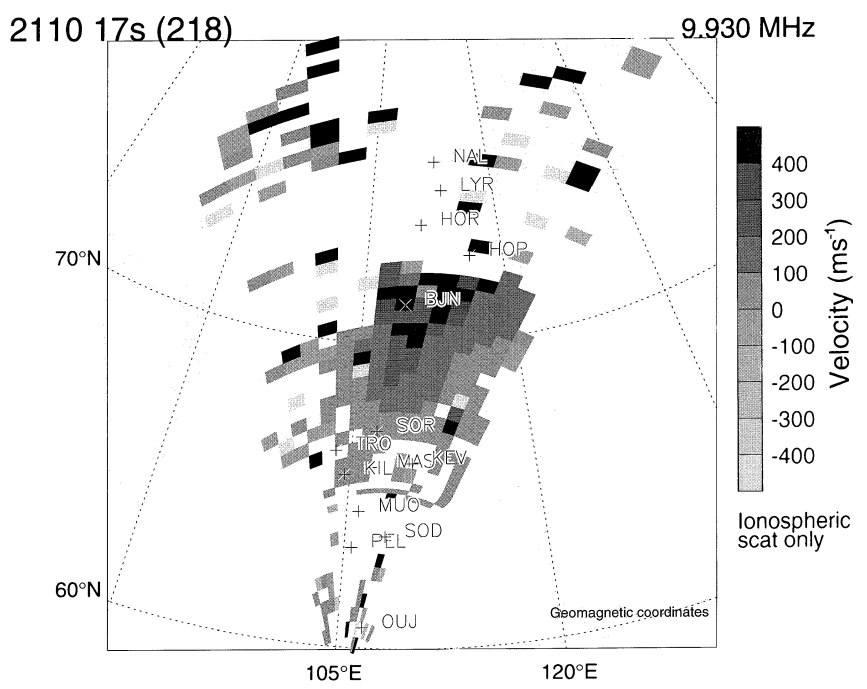

Fig. 5. Line of sight velocity data from a $4 \mathrm{~min}, 16$ beam anticlockwise scan of the CUTLASS Finland radar recorded between 21:10:17-21:14:17 UT, indicating the spatial extent of one of the equatorward velocity pulses shown in Fig. 4. The locations of IMAGE magnetometer stations are also indicated

equatorward velocity pulse displayed in Fig. 4, and thus temporal effects are not preventing an estimation of the apparent spatial extent of the pulse. The pulse can be seen to occupy some 5-6 beams of the CUTLASS scan, corresponding to $400-500 \mathrm{~km}$ in longitude, and some 79 range gates, corresponding to $300-400 \mathrm{~km}$ in latitude, although the pulse is present in the observed backscatter up to its poleward edge, and thus it may well have a greater poleward extent than that measured here. In additional to quantifying the spatial extent of the velocity pulses, the scan data from CUTLASS can, in conjunction with the high time resolution data from beam 9 , reveal any propagation effects in the data. The radar data do not reveal any measurable azimuthal propagation effects in the pulses of ionospheric velocity, rather they appear to form in a given position, grow, and then decay. Azimuthally separated IMAGE stations can give additional information on any propagation of the pulses. Such magnetometer data provide some evidence for an eastward propagation of the features, showing either a small or a zero time delay between azimuthally separated magnetometer stations.

\section{Discussion}

\subsection{HF absorption}

An important feature in the HF radar observations of ionospheric convection during substorms is the periods of reduced backscatter in the intervals 20:07-20:12 UT, 20:27-20:44 UT and 22:02-22:24 UT. These reductions in observed backscatter are the result of $D$ region absorption of the radar's HF radiation as a consequence of enhanced energetic particle precipitation during the 
substorm process (e.g. Milan et al., 1996). In spite of these dropouts, the CUTLASS Finland radar records backscatter throughout the interval of substorm activity, with a substantial band extending over some $890 \mathrm{~km}$ for the majority of the interval under study and a minimum coverage $220 \mathrm{~km}$. The significant losses of HF backscatter are confined to events close enough to the instrumentation for large auroral zone electrojet perturbations to be observed in the nearby ground magnetometers, and occur regardless of whether these currents are centred to the west or east of the instruments. Previously HF radar studies of the substorm expansion phase have been limited as a consequence of this absorption process, with relatively few studies published with an extensive region of HF backscatter (Morelli et al., 1995; Yeoman and Pinnock, 1996). The limited effect of HF absorption in the present study is probably a consequence of the relatively high latitude of the substorm activity recorded here, with the electrojet centre lying between $67^{\circ}$ and $71^{\circ}$ geomagnetic latitude. These high-latitude substorm onsets occur during an interval of quiet magnetic activity, with $K_{p}$ during the interval being 2- and $\Sigma K_{p}$ for the day being only 8-. These magnetic activity conditions have enabled HF backscatter from a region covering $65^{\circ}-73^{\circ}$ geomagnetic under the summer, night time conditions prevailing here.

\subsection{Transient convection features}

The observation of the temporal and spatial extent of transient bursts of equatorward flow in the substorm expansion phase provided by such high time resolution CUTLASS HF radar data is new, although some evidence of bursty flows, associated with substorms, has been seen before (Lester et al., 1995; Morelli et al., 1995). The morphology of the ionospheric flows causing the CUTLASS observations can be further investigated by comparing the convection velocity measurements made by the CUTLASS radar with the magnetic field variations measured by the IMAGE magnetometer stations in the Svalbard region. One likely candidate mechanism is a pattern of vortical ionospheric currents, produced by pairs of upward and downward field aligned currents in the magnetosphere. The magnetic field signature of such a current system for a homogeneous conductivity distribution has been modelled by Wei and Lee (1990) and the plasma streamlines in the ionosphere and the resultant ground magnetic field signature are displayed in Fig. 6. The magnetic field variations associated with the CUTLASS velocity pulse measurements can be identified from the polarities of the changes in the $X$ and $Y$ components of the magnetic field measured in the region of the velocity pulse, such as those presented in Fig. 4. The polarities of the field changes can be compared with those expected from the modelling presented in Fig. 6 . The polarities of the $X$ and $Y$ component magnetic field measurements from the IMAGE stations on Svalbard and in northern Scandinavia are qualitatively consistent with the picture of a

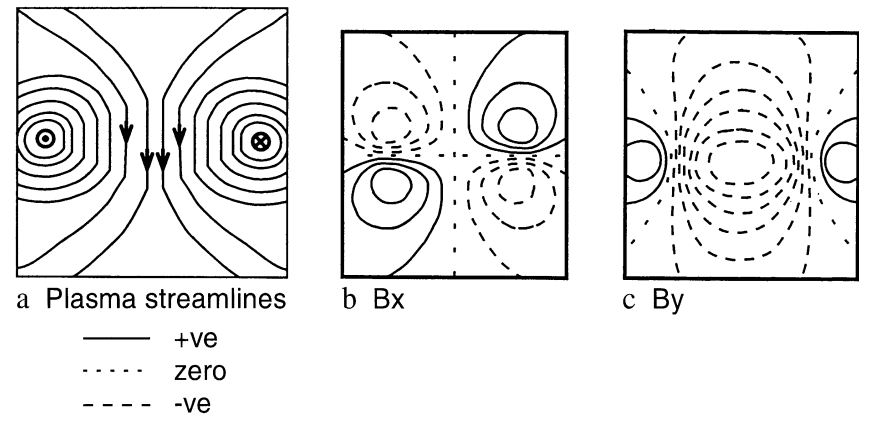

Fig. 6. a Plasma streamlines associated with a pair of field aligned currents, marked by up-arrow and down-arrow symbols. b and $\mathbf{c}$ show the $B_{x}$ and $B_{y}$ magnetic field perturbations associated with the streamlines in a (after Wei and Lee, 1990)

Hall current vortex from a pair of field aligned currents. For the first equatorward flow pulse (observed between 20:50 and 20:54 UT) this current system, based on the IMAGE magnetometer observations, appears to be centred over BJN. The subsequent two pulses are slightly south and east of this. These magnetometer observations are consistent with the locations of the equatorward flows observed with the CUTLASS radar in each case. The full scan data from the CUTLASS radar (Fig. 5) has in addition allowed an estimate of the size of the vortex system to be made, and it appears to be of order $500 \mathrm{~km}$ extent in both latitude and longitude. This interpretation assumes an essentially stationary pair of current vortices growing and fading in the radar field-of-view.

Although the high time resolution beam 9 has enabled a study of the substorm-associated flow transients, a full characterisation of the flow vortex as presented in Fig. 5 is hampered by 4-min full scan period. An estimate of the vortex size can only be attempted when the whole of a radar scan occurs during the velocity pulse and thus temporal effects are not preventing a proper estimation of the spatial extent of the pulse. Even in such a case the monostatic nature of the radar data means that the measurements are dominated by the north-south velocity components. The use of magnetometer equivalent currents, discussed next in this section and illustrated in Fig. 3 and Fig. 7, provide the best indication of the vortical nature of the ionospheric flows.

An alternative interpretation to the sequence of magnetic field and velocity perturbations associated with the transient features is that they are caused by a motion of the current systems, analogous to that of an $\Omega$-band (e.g. Lühr and Schlegel, 1994). Such a motion is suggested by a phase delay of the $Y$ component at HOP of about $40 \mathrm{~s}$ with respect to HOR. An appropriate way to visualise moving transient current features is to plot sequences of equivalent current vectors from a chain of stations which is approximately perpendicular to the direction of motion. For an E region current system with a spatial extent of greater than the $\mathrm{E}$ region height, and assuming a horizontally uniform ionospheric conductivity, then the ground magnetic field deflections, $b$, 


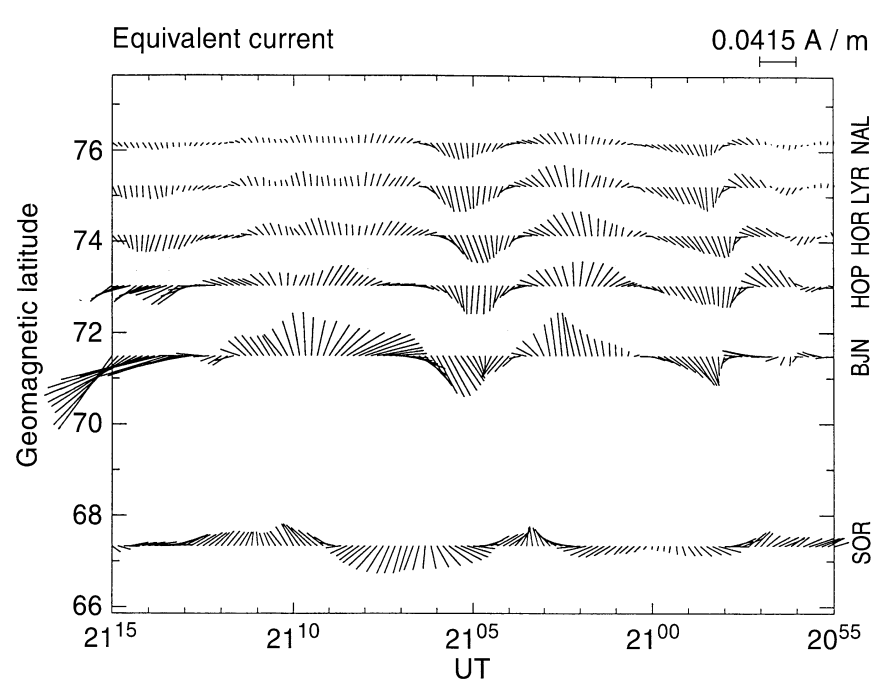

Fig. 7. Equivalent current patterns deduced from IMAGE magnetometer data. The current vectors have been plotted versus an inverted time axis. This is appropriate for visualising eastward moving features. The time series at HOP has been shifted by $40 \mathrm{~s}$ to account for the propagation delay

can be related to an ionospheric equivalent current intensity, $J$, by

$J_{x}=-\frac{2}{\mu_{0}} b_{y} \quad J_{y}=\frac{2}{\mu_{0}} b_{x}$,

where $x$ and $y$ indicate the northward and eastward components respectively. Since the features appear to move eastwards, according to the observed propagation delay, the current vectors have to be plotted against an inverted time scale to give a correct image of the spatial current distribution (see e.g. Lühr and Schlegel, 1994). Figure 7 presents such equivalent current patterns derived from IMAGE stations NAL through SOR for the most active period 20:55 to 21:51 UT. The time series at HOP has been shifted by $40 \mathrm{~s}$ to make it fit the stations along the meridian. During the interval displayed at least five vortices centred near BJN, and showing alternating senses of rotation can be identified: 20:57:30 UT (anticlockwise, ACW), 21:00:30 UT (clockwise, CW), 21:03:30 UT (ACW), 21:07:10 UT (CW) and 21:11:30 UT (ACW). Clockwise and anticlockwise current vortices are associated with downward and upward FACs respectively (Fig. 6).

In the case of a time-stationary current system moving overhead, temporal variations can converted into spatial scales. The deduced time delay between HOR and HOP implies an eastward propagation speed of $\leq 6 \mathrm{~km} \mathrm{~s}^{-1}$, depending on the orientation of the current front. Since the vortex centres are separated by $\sim 3 \mathrm{~min}$, this converts to a distance between antiparallel FAC filaments of $\leq 1800 \mathrm{~km}$. This derived separation is significantly larger than the width of the high flow region determined by the CUTLASS radar. This is likely to be due in part to the effect of the CUTLASS scanning mode. The CUTLASS Finland scan during this interval ran from east to west. If the current vortex features propagate eastwards, then the observed longitudinal extent is given by

$\lambda=\lambda_{c}\left(1+\frac{v_{\text {feature }}}{v_{\text {scan }}}\right) \cong 1000 \mathrm{~km}$

where $\lambda_{c}=500 \mathrm{~km}$ is the width of the feature deduced by CUTLASS, $v_{\text {feature }}=6 \mathrm{~km} \mathrm{~s}^{-1}$ is the eastward velocity of the current features and $v_{\text {scan }}=5.5 \mathrm{~km} \mathrm{~s}^{-1}$ is the velocity of the CUTLASS scan at the latitude of BJN. With this correction both estimates become more consistent. Unfortunately the propagation cannot be confirmed by the azimuthally separated IMAGE stations TRO and KEV further south. This may be a consequence of the vortex feature being smaller at these latitudes and thus these magnetograms being dominated by other effects. The observed signature may well actually arise from some combination of growth/fading and propagation.

An examination of the line of sight velocity from a single range-beam cell during the impulsive convection features presented in the top panel of Fig. 4, in addition to clearly demonstrating the equatorward pulses (positive Doppler velocity) also reveals a suppression of flow prior to each burst of flow for pulses 1, 3, 4 and 5. The morphology, duration, repetition rate and spatial extent of these features are very similar to the impulsive electrojet signatures seen with the PACE radar at Halley, Antarctica, by Morelli et al. (1995). Morelli and coworkers interpreted these reductions in flow as being a consequence of very high ionospheric conductivity due to particle precipitation during the substorm cycle, with the subsequent equatorward pulses occurring when the conductivity dropped after precipitation halted.

The impulsive vortex features described occur during a period of equatorward flow of $\sim 200 \mathrm{~m} \mathrm{~s}^{-1}$, and follow on 18-70 min after the substorm expansion phase onset. There is no evidence of Pi2 pulsation activity accompanying the features, unlike the observations of Morelli et al., (1995) where the majority of impulsive features were accompanied by $\mathrm{Pi} 2$ waves.

The relationship between the ionospheric velocity (and hence the electric field) and the ground magnetic field can be examined further by combining the deduced equivalent current intensities with the ionospheric velocities. As the equivalent Hall currents $J_{H}$ are related to the ionospheric electric field, $E$, and hence the plasma convection velocity, $v$, via the height integrated Hall conductance, $\sum_{H}$, by

$J_{H}=\sum_{H} E=\sum_{H} v B$,

where $B$ is the magnetic field strength, in the present experimental arrangement an estimate of the Hall conductance may be calculated from (Lühr et al., 1993, 1996).

$\sum_{H} \approx-\frac{2}{\mu_{0}} \frac{b_{y}}{v_{x} B}$.

Figure $3 b$ displays the beam 9 line-of-sight velocities, represented as a colour scale, as a function of magnetic 
latitude. The equivalent current intensities derived from the magnetic field data from SOR, BJN and HOP are overlaid at the magnetic latitude appropriate for each station. The magnetic field data has been high-pass filtered at $1200 \mathrm{~s}$ to remove the background field before calculating the equivalent current intensities. The upper panel presents an estimate of the Hall conductance, derived from the BJN magnetic field, again high-pass filtered at $1200 \mathrm{~s}$ and the CUTLASS Finland line-ofsight velocity from the overlying range gate.

A number of interesting features can be seen in Fig. 3b. The intervals of enhanced equatorward flows clearly correlate with strong poleward Hall currents, as expected. The $Y$ component equivalent currents provide additional information about the azimuthal flow in the ionosphere, which was not available from the line-ofsight data from the CUTLASS Finland high time resolution beam. These equivalent current measurements confirm the vortical nature of the flow transients. It is also interesting to note that the flow suppressions preceding the flow enhancements represent intervals of considerable (equatorward) currents. This high current, low electric field region is reflected in the deduced Hall conductance measurements (upper panel of Fig. 3b), which show enhancements in the Hall conductance of 10-40 S. The background Hall conductance derived from the present technique is largely dependent on the background magnetic field, which is difficult to determine exactly. Hence these Hall conductance measurements are probably best regarded as enhancements on the background $\mathrm{E}$ region Hall conductance, rather than absolute measurements of it. The shaded bars in the upper panel of Fig. $3 \mathrm{~b}$ represent intervals when the calculation of $\sum_{H}$ broke down, leading to nonsensical results. These intervals coincide with the beginnings of the high conductivity conditions, and are likely to be a consequence of strong conductivity gradients associated with particle precipitation. The breakdown of the technique does illustrate the limitations of the technique employed, and measurements of this type will soon be enhanced in this region by the EISCAT Svalbard incoherent scatter radar. A careful comparison of the magnetic field and radar data reveals that there is a systematic phase difference between the ionospheric flows and the magnetic field deflections. This can be seen in Fig. 4 where the negative deflections of the BJN $Y$ component lead the flowbursts on average by some $70 \mathrm{~s}$, which corresponds to $17 \%$ of the cycle time. Considering the earlier mentioned eastward propagation of the features this means that the high equatorward flow velocities are located some $400 \mathrm{~km}$ west of the peaks in poleward equivalent current. Such displacement between flows and current is another indicator of ionospheric conductivity gradients. In such a situation equivalent currents cannot be simply regarded as Hall currents, but rather a more rigorous treatment such as that of Glassmeier (1987) has to be applied in order to deduce the ionospheric currents and conductivities. The full analysis of these quantities is, however, beyond the scope of this study.

\subsection{Relationship to magnetotail phenomena}

The dynamic variations of ionospheric electric field and currents and localised FAC systems presented here clearly have important implications for the energetics of magnetosphere-ionosphere coupling during substorms. They may also have implications for the substorm onset process in the magnetotail. Although there are a number of competing substorm onset theories (see e.g. Lui, 1991) one of the critical issues of recent years common to all has been the location of the substorm onset region. Debate on this issue has centred around two substorm onset theories. The near Earth neutral line (NENL) theory is a longstanding theory of substorm onset (Russell and McPherron, 1973; Hones 1979) which proposes the neutral line onset region to be located at $>20 R_{E}$ downtail (see e.g. Baker et al., 1996 for a recent review). The current disruption model is a more recent theory which, in contrast, puts onset at a distance of 6-8 $R_{E}$ (see e.g. Lui, 1996). There is a considerable body of recent evidence that substorm onset occurs in the very near Earth region, subsequently propagating tailward (Lopez and Lui, 1990; Lopez et al., 1993), with reconnection following in the more distant magnetotail. In a case study, Yeoman et al. (1994) identified substorm onset at $\sim 7 R_{E}$. In a subsequent study of the same interval Yeoman and Pinnock (1996) identified the ionospheric signatures of magnetotail reconnection some 27 min later, implying an X line location of 43 $R_{E}$. In a statistical study of substorm onset and polar cap dynamics Taylor et al. (1996) calculated typical delays of 20-50 min between substorm onset and the destruction of open flux, corresponding to NENL locations of 35-75 $R_{E}$. However other recent studies have suggested that NENL reconnection is the process which initiates substorm onset, with the near-Earth signatures a consequence of the more distant tail process (e.g. Baker et al., 1993).

The duration, repetition rate and morphology of the impulsive features presented here is very similar to that of flux transfer events on the dayside (e.g. Lockwood et al., 1990), and this might suggest that the source mechanism is transient reconnection at the NENL. If these features are signs of reconnection they occur some 17-70 min after the substorm expansion phase onset and the transient ionospheric features may have been produced by earthward flowbursts in the tail such as those reported by e.g. Baumjohann et al. (1990), and Angelopoulos et al. (1992). Magnetotail flowbursts have a typical time scale of $\sim 1 \mathrm{~min}$ and occur in bursty intervals of typical duration $\sim 10 \mathrm{~min}$. The ionospheric vortex observations presented here have a time scale between these two limits. Impulsive current disruption in the nearer-Earth tail region could also produce the observed features, although they would be expected to be associated with near-Earth magnetic field dipolarisation and hence Pi2 pulsations.

A sequence of ionospheric convection vortices of alternating sense of rotation, whose motion is largely directed azimuthally rather than meridionally, has been 
observed. The latitude of convection vortices created by velocity shears in the magnetotail would be expected to lie poleward of the electrojet, whereas the observations presented here lie just poleward of the centre of the electrojet. This suggests that the observed ionospheric convection vortices may be features created by the magnetotail flowbursts rather than corresponding directly to them. In spite of these reservations, it is possible to calculate the magnetotail parameters implied by the ionospheric observations and compare them to those found in magnetotail bursty bulk flows (BBFs). Mapping using the Tsyganenko T89 magnetic field model for $K_{p}=1$ (see Tsyganenko, 1990) suggests that the vortical features map some $40-50 R_{E}$ downtail (although there is considerable uncertainty in this figure, and T89 is known to overestimate this parameter), and occupy a region of $\sim 12 R_{E}$ in the $Y$ direction. Most BBF observations have been made at $\sim 20 R_{E}$ and have suggested a $Y$ extent of $\sim 10 R_{E}$ or less. If the vortical features represent a loss of open flux due to tail reconnection then the measured voltage associated with the vortices, of $\sim 30 \mathrm{kV}$, can be calculated, assuming a spherical polar cap, using the method of Lockwood and Cowley (1992) to represent a flux closure rate of $\sim 3 \times 10^{4} \mathrm{~Wb} \mathrm{~s}^{-1}$, representing $\sim 7 \times 10^{6} \mathrm{~Wb}$ per flowburst and or $\sim 2 \times 10^{7} \mathrm{~Wb}$ for the sequence of vortices. This is similar to that estimated during BBFs by Angelopoulos et al. (1992) and compares with a rate of loss of open flux of $5 \times 10^{5} \mathrm{~Wb} \mathrm{~s}^{-1}$ measured during the expansion phase using the AMIE technique by Taylor et al. (1996). In the study of Taylor et al. (1996) this rate of flux closure corresponded to a flux loss of $\sim 10^{9} \mathrm{~Wb}$ during the expansion phase, as a result of a number of substorm intensifications during an active magnetic storm period.

\section{Summary}

During the interval 19:00-24:00 UT the CUTLASS Finland radar moved from the return flow region of the dusk convection cell, through the convection reversal region, where relatively slow antisunward flow was observed for around two hours from 20:30 UT, through to 22:30 UT, and into the return flow region of the dawn convection cell. During this interval eight substorm onset/intensifications occurred. For three intervals during the period under study, a reduction in the spatial extent of radar backscatter occurred. The limited effect of HF absorption in the present study is probably a consequence of the high latitude of the substorm activity, with the electrojet centre lying between $67^{\circ}$ and $71^{\circ}$ geomagnetic latitude during the interval. These high latitude substorm onsets occur during an interval of quiet magnetic activity, with $K_{p}$ during the interval being 2- and $\Sigma K_{p}$ for the day being only 8-. These magnetic activity conditions have enabled HF backscatter to be recorded from a region covering $65^{\circ}-73^{\circ}$ geomagnetic latitude.

During the period 20:40 UT to 21:40 UT, an interval in the expansion phase of a substorm onset, slow equatorward flow of less than $200 \mathrm{~m} \mathrm{~s}^{-1}$ was observed over most of the field of view of the CUTLASS radar. This interval was punctuated by short intervals of suppressed flow, followed by the observation of pulses of more intense equatorward ionospheric flows, exceeding $\sim 600 \mathrm{~m} \mathrm{~s}^{-1}$. These pulses had a duration of 3-5 min and a repetition period of $\sim 8 \mathrm{~min}$. Their spatial extent in the CUTLASS field of view was $400-500 \mathrm{~km}$ in longitude, and $300-400 \mathrm{~km}$ in latitude. They are interpreted as signatures of ionospheric current vortices associated with pairs of field aligned currents, with their apparent spatial and temporal extent probably caused by a combination of growth/fading and propagation.

Each pulse of enhanced equatorward flow was preceded by an interval of suppressed flow and enhanced ionospheric Hall conductance. This sequence is consistent with the signature expected from a pair of field aligned currents. An interval of enhanced energetic particle precipitation associated with an upward field aligned current would cause an increase in electron density and hence ionospheric Hall conductance, with current continuity then requiring a reduction in the ionospheric electric field and drift velocity. Recombination then lowers the conductance, and a strong ionospheric flow is observed, in the form of a pair of current vortices.

In the ionospheric electric field and ground magnetic field signatures presented here, the distribution of magnetometer stations, and the CUTLASS high time resolution data have enabled a determination of the morphology of the impulsive electrojet features. The relationship between these observations and substorm phenomena in the magnetotail remains open to debate, as does the implication for the near-Earth neutral line (NENL) and current disruption models for substorm expansion phase onset.

HF radars have been demonstrated to be very valuable tools for high time resolution studies of the ionospheric convection signatures of substorms. The transient features revealed demonstrate the highly dynamic nature of current, conductivity and electric field, observations which have important implications for the energetics of magnetosphere-ionosphere coupling and magnetospheric onset region of the substorm. The HF radars and ground-based magnetometer arrays have provided highly complementary datasets which have helped to resolve the ambiguities inherent in such very dynamic phenomena.

Acknowledgements. We would like to thank Dr Dave Milling, University of York, for providing the SAMNET data. The CUTLASS HF radars are deployed and operated by the University of Leicester, and are jointly funded by the UK Particle Physics and Astronomy Research Council, the Finnish Meteorological Institute, and the Swedish Institute for Space Physics.

Topical Editor D. Alcayde thanks R. E. Lopez and J. P. Villain for their help in evaluating this paper.

\section{References}

Angelopoulos, V., W. Baumjohann, C. F. Kennel, F. V. Coroniti, M. G. Kivelson, R. Pellat, R. J. Walker. H. Lühr, and 
G. Paschmann, Bursty bulk flows in the inner central plasma sheet, J. Geophys. Res., 97, 4027-4039, 1992.

Baker, K. B., and S. Wing, A new magnetic coordinate system for conjugate studies at high latitudes, J. Geophys. Res., 94, 91399143, 1989.

Baker, D. N., T. I. Pulkkinen, R. L. McPherron, J. D. Craven, L. A. Frank, R. D. Elphinstone, J. S. Murphree, J. F. Fennel, R. E. Lopez, and T. Nagai, CDAW 9 analysis of magnetospheric events of May 3, 1986: event C, J. Geophys. Res., 98, 3815-3834, 1993.

Baker, D. N., T. I. Pulkkinen, V. Angelopoulos, W. Baumjohann, and R. L. McPherron, Neutral line model of substorms: past results and present view, J. Geophys. Res., 101, 12, 975-13,010, 1996.

Baumjohann, W., R. J. Pellinen, H. J. Opgenoorth, and E. Nielsen, Joint two-dimensional observations of ground magnetic and ionospheric electric fields associated with auroral zone currents: current systems associated with local auroral break-ups, Planet. Space. Sci., 29, 431-447, 1981.

Baumjohann, W., G. Paschmann, and H. Lühr, Characteristics of high-speed ion flows in the plasma sheet, J. Geophys. Res., 95, 3801-3809, 1990.

Glassmeier, K.-H., Ground-based observations of field-aligned currents in the auroral zone: Methods and results, Ann. Geophysicae, 5, 115-126, 1987.

Greenwald, R. A., K. B. Baker, J. R. Dudeney, M. Pinnock, T. B. Jones, E. C. Thomas, J.-P. Villain, J. -C. Cerisier, C. Senior, C. Hanuise, R. D. Hunsucker, G. Sofko, J. Koehler, E. Nielsen, R. Pellinen, A. D. M. Walker, N. Sato, and H. Yamagishi, Darn/ Superdarn: a global view of the dynamics of high-latitude convection, Space Science Reviews, 71, 761-796, 1995.

Hones, E. W. Jr., Transient phenomena in the magnetotail and their relation to substorms, Space Sci. Rev., 23, 393-410, 1979.

Inhester, B., W. Baumjohann, R. A. Greenwald, and E. Nielsen, Joint two-dimensional observations of ground magnetic and ionospheric electric fields associated with auroral zone currents 3. Auroral zone currents during the passage of a westward travelling surge, J. Geophys.,49, 155-162, 1981.

Kirkwood, S., H. J. Opgenoorth, and J. S. Murphree, Ionospheric conductivities, electric fields and currents associated with auroral substorms measured by the EISCAT radar, Planet. Space Sci., 36, 1359-1380, 1988.

Kisabeth, J. L., and G. Rostoker, Current flow in auroral loops and surges inferred from ground-based magnetic observations, $J$. Geophys. Res., 78, 5573-5584, 1973.

Lester, M., W. J. Hughes, and H. J. Singer, Polarization patterns of $\mathrm{Pi} 2$ magnetic pulsations and the substorm current wedge, $J$. Geophys. Res., 88, 7958-7966, 1983.

Lester, M., M. Lockwood, T. K. Yeoman, S. W. H. Cowley, H. Lühr, R. Bunting, and C. J. Farrugia, The response of ionospheric convection in the polar cap to substorm activity, Ann. Geophysicae, 13, 147-158, 1995.

Lockwood, M. and S. W. H. Cowley, Ionospheric convection and the substorm cycle, Proceedings of ICS-I, ESA SP-335, 99-109, 1992.

Lockwood, M., S. W. H. Cowley, P. E. Sandholt, and R. P. Lepping, The ionospheric signatures of flux transfer events and solar wind dynamic pressure changes, J. Geophys. Res., 95, 17, 113135, 1990.

Lopez, R. E., and A. T. Y. Lui, A multisatellite case study of the expansion of a substorm current wedge in the near-Earth magnetotail, J. Geophys., Res., 95, 8009-8017, 1990.

Lopez, R. E., H. E. J. Koskinen, T. I. Pulkkinen, T. Bösinger, R. W. McEntire, and T. A. Potemra, Simultaneous observation of the poleward expansion of substorm electrojet activity and the tailward expansion of current sheet disruption in the near-Earth magnetotial, J. Geophys. Res., 98, 9285-9295, 1993.

Lühr, H., The IMAGE magnetometer network, STEP International Newsletter, 4, (10), 4-6, 1994.
Lühr, H., and K. Schlegel, Combined measurements of EISCAT and the EISCAT magnetometer cross to study $\Omega$ bands, $J$. Geophys. Res., 99, 8951-8959, 1994.

Lühr, H., W. Blawert, and H. Todd, The ionospheric plasma flow and current patterns of travelling convection vortices: a case study, J. Atmos. Terr. Phys., 55, 1717-1727, 1993.

Lühr, H., M. Lockwood, P. E. Sandholt, T. L. Hansen, and T. Moretto, Multi-instrument ground-based observations of a travelling convection vortices event, Ann. Geophysicae, 14, 162$181,1996$.

Lui, A. T. Y., A synthesis of magnetospheric substorm models, $J$. Geophys. Res., 96, 1849-1856, 1991.

Lui, A. T. Y., Current disruption in the Earth's magnetosphere: observations and models, J. Geophys, Res., 101, 13,067-13,088, 1996.

Milan, S. E., T. B. Jones, M. Lester, E. M. Warrington, and G. D. Reeves, Substorm correlated absorption on a $3200 \mathrm{~km}$ transauroral HF propagation path, Ann. Geophysicae, 14, 182-190, 1996.

Morelli, J. P., R. J. Bunting, S. W. H. Cowley, C. J. Farrugia, M. P. Freeman, E. Friis-Christensen, G. O. L. Jones, M. Lester, R. V. Lewis, H. Lühr, D. Orr, M.Pinnock, G. D. Reeves, P. J. S. Williams, and T. K. Yeoman, Radar observations of auroral zone flows during a multiple-onset substorm, Ann. Geophysicae, 13, 1144-1163, 1995.

Opgenoorth, H. J., R. J. Pellinen, W. Baumjohann, E. Nielsen, G. Marklund, and L. Eliasson, Three dimensional current flow and particle precipitation in a westward travelling surge (observed during the Barium-GEOS rocket experiment), $J$. Geophys. Res., 88, 3138-3152, 1983.

Pinnock, M., A. S. Rodger, J. R. Dudeney, F. Rich, and K. B. Baker, High spatial and temporal resolution observations of the ionospheric cusp. Ann. Geophysicae, 13, 919-925, 1995.

Robinson, T. R., Towards a self-consistent non-linear theory of radar-auroral backscatter, J. Atmos. Terr. Phys., 48, 417-422, 1986.

Rostoker, G., S.-I. Akasofu, J. Foster, R. A. Greenwald, Y. Kamide, K. Kawasaki, A. T. Y. Lui, R. L. McPherron, and C. T. Russell, Magnetospheric substorms - definition and signatures, $J$. Geophys. Res., 85, 1663-1668, 1980.

Russell, C. T., and R. L. McPherron, The magnetotail and substorms, Space Sci. Rev., 15, 205-266, 1973.

Samson, J. C., and G. Rostoker, Polarization characteristics of Pi2 pulsations and implications for their source mechanisms: influence of the westward travelling surge, Planet. Space Sci., 31, 435-458, 1983.

Taylor, J. R., T. K. Yeoman, M. Lester, B. A. Emery, and D. J. Knipp, Variations in the polar cap area during intervals of substorm activity on March 20-21, 1990 deduced from AMIE convection patterns, Ann. Geophysicae, 14, 879-887, 1996.

Tsyganenko, N. A., Quantitative models of the magnetospheric magnetic field: methods and results, Space Sci. Rev., 54, 75-186, 1990.

Wei, C. Q. and L. C. Lee, Ground magnetic signatures of moving elongated plasma clouds, J. Geophys. Res., 95, 2405-2418, 1990.

Yeoman, T. K., and M. Pinnock, The high-latitude convection response to an interval of substorm activity, Ann. Geophysicae, 14, 518-532, 1996.

Yeoman, T. K., D. K. Milling, and D. Orr, Pi2 pulsation polarization patterns on the U.K. Sub-auroral Magnetometer Network (SAMNET), Planet. Space Sci. 38, 589-602, 1990.

Yeoman, T. K., M. Lester, D. K. Milling and D. Orr, Polarization, propagation and MHD wave modes of $\mathrm{Pi} 2$ pulsations: SABRE/ SAMNET results, Planet. Space Sci., 39, 983-998, 1991.

Yeoman, T. K., H. Lühr, R. W. H. Friedel, S. Coles, M. Grandé, C. H. Perry, M. Lester, P. N. Smith, H. J. Singer, and D. Orr, CRRES/Ground-based multi-instrument observations of an interval of substorm activity, Ann. Geophysicae, 12, 1174-1191, 1994. 